\title{
ESPACIOS NATURALES DE RIBERA EN EL MUNICIPIO DE ZARAGOZA
}

\author{
Alfredo OLLERO OJEDA \\ Universidad de Zaragoza.
}

\begin{abstract}
RESUMEN: El término municipal de Zaragoza es recorrido por los ríos Ebro, Gállego y Huerva, cuyas riberas cuentan con un total de 606 Has. que podrian ser catalogadas como espacios naturales. Se lleva a cabo un estudio de ordenación del medio natural exponiendo el interés de los espacios ribereños zaragozanos, sus características, su localización, el diagnóstico de su situación actual y los impactos que sufren, concluyéndose con el planteamiento de alternativas.
\end{abstract}

ABSTRACT: Zaragoza city is crossed by the rivers Ebro, Gállego \& Huerva, whose banks count on a total of 606 Has., which might be thought of as being natural spaces. A research about ordenation in a natural means is undergone, taking into account the interest of riverside spaces in Zaragoza, their characteristics, location, the diagnosis of their situation nowedays and the resulting impacts, concluding with the setting of alternatives.

Sumario: Introducción.- Características generales de las riberas zaragozanas.- Los sotos del Ebro.- Las riberas del Gállego.- Las riberas de la Huerva.- Diagnóstico.- Impactos.Alternativas.- Bibliografía.

\section{INTRODUCCION}

El presente trabajo es resultado de investigaciones llevadas a cabo para la realización de un inventario de espacios naturales del municipio de Zaragoza (ANTON et al., 1990), en el que el autor ha elaborado los espacios de ribera de los ríos Ebro, Gállego y Huerva. Se trata, por tanto de un estudio de ordenación territorial del medio natural que se estructura en varias partes bien definidas: interés y características de los espacios de ribera, localización de los espacios de ribera zaragozanos, diagnóstico de su situación, impactos y planteamiento de alternativas. 
Los bosques de ribera o sotos son ecosistemas de indudable valor, tanto desde el punto de vista ecológico como por su papel en la dinámica fluvial. Su importancia ecológica se ve especialmente revalorizada en la actualidad a causa del carácter relicto de los sotos. En nuestra región, y más concretamente en el término municipal zaragozano, contrastan marcadamente respecto a la aridez circundante, constituyendo enclaves privilegiados para la fauna y de recreo para el hombre. El notable gradiente ecológico de los sotos propicia una riqueza biológica incomparable, dándose la máxima variedad de comunidades vegetales y animales en un espacio reducido.

Pero el principal papel de los bosques de ribera radica en frenar la fuerza de los caudales de crecida e impedir la erosión de las orillas, de manera que el entramado de las raíces de un soto bien desarrollado y conservado constituye la defensa más efectiva contra la erosión fluvial, siendo igualmente el sistema de contención que menos cuidado y mantenimiento requiere.

Un bosque de ribera en situación de avenida aumenta la rugosidad de la orilla, generando turbulencias locales que dispersan la fuerza de la corriente y favorece la sedimentación diferencial de gravas, arenas y limos, formando un suelo aluvial muy rico.

Todo lo expuesto ofrece argumentos más que suficientes para considerar los sotos que aún perviven en las riberas de los ríos que surcan el término de Zaragoza como un conjunto de espacios naturales de interés máximo, cuyos valores ecológicos y recreativos deben ser contemplados y aprovechados en toda actuación municipal.

\section{CARACTERISTICAS GENERALES DE LAS RIBERAS ZARAGOZANAS}

El municipio de Zaragoza cuenta con 606 Has. de sotos y riberas susceptibles de ser considerados espacios naturales. 415 Has. corresponden a las riberas del Ebro, 167 Has. a las del Gállego y 24 Has. a las de la Huerva.

Situados entre el cauce de los ríos y las huertas, destacan desde el punto de vista microclimático por su importante humedad ambiental. El ambiente fresco y el sombreado son muy positivos para la fauna terrestre y acuática en época estival, mientras la vegetación de ribera resguarda estos espacios ribereños de la acción del viento. Tanto en valores térmicos como de humedad, las riberas presentan un marcado contraste 
respecto a las huertas próximas y a los sectores áridos algo más alejados. Son frecuentes en invierno las inversiones térmicas con nieblas.

Desde el punto de vista hidrológico destacan las variaciones estacionales de caudal. Ebro y Huerva presentan aguas altas en invierno, con máximo en febrero-marzo, y un estiaje bastante marcado entre finales de junio y comienzos de octubre. El Gállego, por su origen pirenaico, presenta aguas altas primaverales. Dada la inclusión de los sotos en el cauce mayor y fuera de las motas de contención, la inundación de amplios sectores de los mismos es frecuente, siendo raro el invierno que no se produce. Ello implica unas variaciones estacionales muy marcadas en estos espacios, que afectan tanto a aspectos puramente perceptuales como a las posibilidades de actuación sobre los mismos, incluyendo la propia posibilidad de acceso.

Desde el punto de vista geomorfológico, los tres ríos representados constituyen otros tantos modelos de cauce:

-El Ebro describe meandros libres y se desgaja en algunos puntos en brazos secundarios, brazos ciegos, galachos... Su cauce dinámico ha experimentado notables variaciones en su labor continua de creación, exageración, migración y corta de meandros, a causa de las crecidas del río. La llanura de inundación del río Ebro, sobre la que se asientan los sotos, presenta una anchura en la zona de estudio que oscila entre los 3 y $5 \mathrm{~km}$. Su planitud se encuentra alterada por complicadas microtopografías (a lo sumo $2 \mathrm{~m}$ ) resultantes de la sedimentación diferencial (gravas, arenas, limos y arcillas) de los materiales arrastrados por las crecidas. Estas microtopografías adquieren importancia a la hora de la distribución de las formaciones vegetales.

- El Gállego transporta una carga de sedimentos muy importante y con abundancia de gravas, lo cual origina un cauce dinámico, de gran anchura en algunos tramos, que combina los modelos trenzado y meandriforme.

-La Huerva describe meandros encajados ( 2 a 4 metros) con un índice de sinuosidad dentro del municipio zaragozano de 1,53. La anchura del cauce no sule superar los 10 metros. 
Por lo que respecta a la vegetación y fauna, los ecosistemas de ribera son enormemente ricos por su amplio gradiente ecológico. Sin entrar en detalles, ya que el tema excedería las dimensiones de este artículo, citar las comunidades vegetales de ribera como carrizales, tamarizales, saucedas, alamedas, choperas, olmedas y pastizales, base de una variada fauna de mamíferos, reptiles, anfibios e invertebrados, sin olvidar la ictiofauna y destacando especialmente la rica y densa avifauna, tanto sedentaria como de paso.

En lo referente a los usos del suelo, los sotos han sido tradicionalmente tierras comunales empleadas para el pastoreo ovino y la entresaca de leña y madera. Las variaciones del cauce originaban frecuentes litigios entre municipios vecinos, que veían alterados sus límites administrativos. En este sentido, puede observarse los límites del municipio zaragozano que se marcaron sobre el cauce del Ebro no coinciden en la actualidad con el mismo en absoluto. Ello es particularmente espectacular en los límites con Utebo y Pastriz.

Actualmente pervivie en bastante menor medida el pastoreo, predominando los cultivos intensivos de huerta para los que se ha ganado espacio progresivamente en detrimento de los sotos. Otros usos son la construcción de obras de contención, la extracción de áridos, la pesca y otros usos recreativos.

Por lo que respecta a los valores perceptuales, si bien la amplitud panorámica es reducida, la luminosidad no muy alta, los olores a veces desagradables por la presencia de basuras o de agua detenida y la abundancia de insectos en verano, los espacios de ribera resultan de gran atractivo para el habitante urbano. Ello se debe a la abundancia de sombra, al frescor ambiental, a la tranquilidad de los sonidos y a la presencia de láminas de agua, lo cual incide en un grado de bienestar alto que revaloriza la demanda recreativa de estos espacios.

\section{LOS SOTOS DEL EBRO (mapas 1 y 2 )}

En ambas márgenes del río Ebro, aguas arriba y abajo de la ciudad de Zaragoza, se conservan 15 espacios como últimos restos de la vegetación que antaño cubría por 
completo las riberas. Ordenados desde aguas arriba hacia aguas abajo, han sido numerados para su localización en los mapas.

1) Soto de Mejana Redonda (30TXM675226). Se localiza al pie del escarpe de yesos Remolinos-Juslibol, sobre una antigua flecha lateral separada de la pared por un brazo ciego. Se trata de un lugar prácticamente inaccesible, lo cual ha permitido su magnífico estado actual, de manera que se plantea como reserva integral. El soto ( 3 Has.) ha alcanzado sus formaciones climácicas (sauceda de orla y alameda), destacando la existencia de una colonia de tamarices adosados al brazo ciego, al pie del escarpe, debido a la salinidad del lugar.

2) Soto de Alfocea (30TXM700199). Cuenta con una superficie de 15 Has, no todas de arbolado, y está muy degradado por los visitantes, teniendo en cuenta la facilidad del acceso. Se trata de un soto abierto con predominio de álamos y un sotobosque empobrecido. Se encuentra adosado a una extensa superficie de gravas en fase de colonización vegetal sobre la que son evidentes las huellas de antiguas extracciones. Dada la localización del espacio y su degradación ecológica irreversible, puede plantearse como zona recreativa con el fin de atraer visitantes potenciales de otros espacios de mayor valor.

3) Galacho de Juslibol y Soto de Partinchas (30TXM722193). Con una superficie global de 115 Has. constituye sin lugar a dudas el espacio de ribera de mayor valor del municipio zaragozano. El Galacho de Juslibol constituye un meandro recientemente abandonado por el río Ebro, en concreto en la crecida de enero de 1961, habiendo vivido con posterioridad las sucesivas etapas de colonización biológica. El espacio, al pie del escarpe de yesos, consta de una zona húmeda formada por el propio galacho, cubierto en parte por carrizales, y una serie de lagunas derivadas de la extracción de gravas, además de un soto antiguo muy bien desarrollado, el de Partinchas, que jugó un papel trascendental en la crecida citada. Por la variedad de ecosistemas que reúne, el espacio se encuentra en proceso de estudio de cara al establecimiento de una figura de protección.

4) Soto de Torre Alqué (30TXM712192). Con sólo 12 Has. disponibles y escaso valor ecológico, podría resultar interesante como zona de descongestión del Galacho de Juslibol, situado al otro lado del río. 
5) Soto de Benavén (30TXM732180). Espacio reducido (6 Has.) y mal comunicado situado entre las huertas y el río, en la margen derecha frente al núcleo de Juslibol. El soto está constituido por una franja arbolada adosada a la orilla que alcanza buenas densidades en algunos sectores. Dada su situación, muy interesante para la dinámica fluvial por su papel protector de la margen, y su alejamiento de las vías de comunicación, conviene a corto plazo su mantenimiento en un estado natural, de manera que no sea roturado ni degradado, de cara a una posterior actuación a medio y largo plazo, por su interés futuro como área recreativa de descongestión.

6) Soto de Ranillas o de la Torre de Bergua (30TXM737156). Se sitúa entre el río y el dique que protege la huerta, al inicio de la orilla convexa de la Revuelta de la Almozara. Dada su proximidad al ACTUR puede plantearse como una buena superficie natural (22 Has.) susceptible de convertirse en zona verde de un barrio superpoblado. Ahora bien, este soto presenta varios sectores de gran valor ecológico, entre los que destaca la isla que lo limita por el Norte, aguas abajo del puente de la autopista. Sería preciso establecer una zonificación de usos, teniendo en cuenta además que la extensión del espacio permite una variedad de los mismos. Asi, se cerrarían espacios de mayor valor ecológico mientras por otros sectores del soto se ofrecería la posibilidad del paseo.

7) Soto de la Almozara (30TXM746140). Con 7 Has., muy degradado e incluido en el tejido urbano, su estado de abandono es alarmante pero su interés potencial muy alto. Posiblemente no se haya urbanizado ni convertido en parque porque se inunda con facilidad, pero podría ser integrado en la ciudad atendiendo únicamente a su cuidado continuo y evitando la deposición de basuras, tan abundantes actualmente. Consideramos imprescindible, a la hora de establecer zonas recreativas de paseo sobre estos sotos, el mantener su fisonomía natural, en todo caso añadiendo algún banco o cartel informativo, pero sin diseñar caminos ni islas de césped o arbolado, costumbre muy difundida, que no hacen sino restar atractivo y romper el conjunto, perjudicando el correcto desarrollo de fauna y flora.

8) Arboleda de Macanaz (30TXM768148). Hoy en día ha perdido totalmente su fisonomía de soto para convertirse en un jardín abierto (2,3 Has.).

9) Soto de Cantalobos (30TXM802128) 20 Has. Muy próximo al barrio de Las Fuentes y muy degradado, especialmente en los sectores próximos a su entrada, con abundantes basuras. El bosque de álamos se encuentra más cerrado conforme avanzamos, 
siendo impenetrable en algunas zonas, si bien abundan los sectores herbosos y suavernente ondulados, mucho más visitados y con continuas huellas de hogueras. Dado que se trata de un soto muy visitado, convendría establecer algún tipo de control sobre el mismo, así como acondicionar su entrada como pequeño parque para que el grueso de los visitantes se queden allí y no accedan a sectores más interesantes. El tramo final, aguas abajo del vértice del meandro que ocupa debería ser protegido.

10) Soto de Villarroya (30TXM803104). Poco visitado por su acceso dificultoso, cuenta con una superficie disponible de 24 Has. Hay sectores específicos que deberían protegerse por su interés ecológico, especialmente los más próximas a la orilla, creando reservas integrales cerradas por barreras de zarzas. Destaca en este sentido el extremo meridional del soto, donde penetra un brazo ciego.

11) Soto de las Perlas (30TXM807097). Resulta uno de los más interesantes, presentando pese a su reducido tamaño (8 Has.) ejemplos de todas las fases de colonización vegetal. No en vano, la formación de este soto es reciente, tratándose de una antigua isla de gravas en el centro del cauce, progresivamente colonizada y adosada definitivamente a la margen derecha. En la actualidad queda separado de las huertas por una gran explotación de gravas cuyo impacto paisajístico sobre el soto es enorme, dándose la circunstancia de que en muchos puntos las acumulaciones de cantos sobrantes procedentes de la extracción están inundando materialmente el límite meridional del soto. La vegetación sigue en continuo progreso y en la actualidad está colonizando una gran flecha de gravas que prolonga la antigua isla hacia aguas abajo. Tanto por su interés ecológico como en la dinámica fluvial, sería interesante la protección de este pequeño soto.

12) Sotos de Picatiel (30TXM824098). La margen izquierda del Ebro, entre Picatiel y el galacho de la Alfranca de Pastriz, constituye uno de los enclaves de todo el Ebro medio donde mayor superficie de sotos se conserva, en un estado bastante aceptable. El interés de los sotos de Picatiel (101 Has.) es enorme, no sólo por contar con toda la amplia variedad de formaciones vegetales derivada de la sucesión de fases de colonización, sino también por presentar a su vez todas las fases de degradàción de los bosques climácicos de ribera, contando hacia su interior con superficies en las que se han plantado choperas artificiales o bien se ha intentado el cultivo, progresando después una colonización vegetal propia de fases recesivas. Si a ello unimos la existencia de una 
extensa barra de gravas adosada a la orilla y de un brazo secundario, ciego en estiaje y funcional en aguas altas, que desemboca en pleno vértice del meandro de La Cartuja, reunimos en este espacio una variedad enorme de ecosistemas de ribera. Ello supone que nos encontramos en el sector de ribera más interesante del municipio después del galacho de Juslibol. Planteamos como alternativas a este espacio la protección más amplia posible, la creación de reservas integrales y la visita guiada.

13) Soto de la Cartuja (30TXM831095). Antiguamente de gran belleza, muy apreciado y visitado, sus 42 Has. se encuentran en una situación actual de total degradación, prácticamente irreversible, teniendo en cuenta además que se está construyendo una urbanización de chalés muy próxima. Las talas han derivado a una ausencia casi total de arbolado, encontrándonos ante un soto muy abierto, con predominio de tamarices y sauces de porte arbustivo y extensos prados carentes de sotobosque. Hay suciedad y extracciones y podría ser un buen área recreativa para La Cartuja.

14) Soto de Benedito (30TXM834086). A pesar de su superficie (17 Has.) más reducida que la del resto de los espacios aguas abajo de Zaragoza, el soto de Benedito, al que se accede directamente desde Pastriz, constituye uno de los parajes más atractivos del municipio, encontrándose además en un estado muy bueno en comparación con otros sotos ribereños. Las basuras existen, pero no son abundantes. El soto propiamente dicho es una alameda de densidad y porte muy notables, contando además con una extensa superficie de gravas (lóbulo del meandro) ya en su casi totalidad colonizada por los sauces. Entre el soto y la sauceda una serie de pequeñas zonas inundadas dotan al espacio de gran atractivo visual y ecológico. En torno a dichas lagunas, que se convierten en brazo secundario del río en momentos de aguas altas, se extienden extensos prados de herbáceas que tapizan las superficies onduladas de los depósitos más recientes. Sería muy interesante la protección de este espacio y el control de las visitas estableciendo en todo caso senderos guiados.

15) Galacho de Lierta (30TXM848072). Se trata de un antiguo brazo del ró al pie del escarpe de la terraza superior, margen derecha, funcional hasta la crecida de febrero de 1952 y abandonado en esa fecha. Actualmente quedan inundadas dos zonas, una con aguas someras, alargada al pie del escarpe y otra de forma elíptica más profunda muy frecuentada por pescadores. Al borde de ambas encontramos carrizos, aneas, chopos blancos y negros, olmos y fresnos abundantes, además de tamariz. El espacio llega hasta 
la orilla del río, donde se encuentra la entrada de un brazo ciego. Como en todos los ejemplos de galacho el interés ecológico es muy alto, por lo que proponemos la protección del espacio, restringiendo su uso recreativo a la práctica de la pesca, siempre con los debidos controles para evitar aglomeraciones y estableciendo un aparcamiento alejado.

\section{LAS RIBERAS DEL GALLEGO (mapa 3)}

En las riberas del Gállego se pueden diferenciar hasta 19 espacios de interés desde San Juan de Mozarrifar hasta el límite con el término de San Mateo de Gállego. Se trata de un sector importante de ribera sin cultivar, no cubierto en su totalidad por sotos, con muchos puntos de elevada degradación. En conjunto supone 311 Has., de las que sólo 167 Has. corresponden al municipio de Zaragoza, dado el carácter limítrofe del cauce con Villanueva de Gállego, Zuera y San Mateo. Es muy de destacar la existencia de este pasillo fluvial casi continuo teniendo en cuenta la alteración total de cauce y riberas que se ha llevado a cabo en los últimos años en el tramo más bajo del curso del Gállego, desde San Juan hasta su desembocadura.

Al Sur de la Cartuja de Aula Dei encontramos tres espacios muy alterados por extracciones de gravas pero de gran valor potencial recreativo, por su proximidad a San Juan de Mozarrifar y Montañana. La vitalidad de la colonización vegetal haría viable la rápida transformación del espacio.

Entre el río y la Cartuja, un pequeño soto prácticamente virgen debería ser urgentemente protegido. En la margen opuesta, la derecha, encontramos otro soto con buen aspecto general, contribuyendo al elevado valor paisajístico de este tramo del río.

Hasta Peñaflor se suceden los espacios en cada lóbulo de meandro, encontrándose en mejor estado los de la margen derecha, correspondientes en su mayor parte al término de Villanueva. Los de la izquierda son muy visitados por la proximidad de la carretera que lleva a Peñaflor, destacando en este sentido la Peña del Cuervo, escarpe vertical sobre el río donde anida abundante avifauna. Los escombros son abundantes desde aqur hasta la localidad de Peñaflor.

Al Norte de dicho núcleo encontramos espacios heterogéneos pero con rincones puntuales de gran interés. El mejor conservado se encuentra marcado en el mapa 3 con la 
letra "R". Muestra toda la gradación ecológica propia de la vegetación de ribera, con una adecuada distribución en franjas desde la orilla del cauce, ofreciendo el mejor ejemplo de soto del bajo Gállego. Por su tamaño ( 30 Has.) permite una zonificación de usos con la posibilidadde establecer áreas de reserva integral y otras recreativas.

\section{LAS RIBERAS DE LA HUERVA}

Constituyen un espacio arbolado casi continuo, desde el límite municipal con Cuarte hasta los pinares del Parque Primo de Rivera, a modo de bosque galería muy estrecho y degradado, de valores naturales poco espectaculares. La franja arbolada aparece en ambas márgenes pero no siempre es accesible con facilidad. El sector más conocido es la Fuente de la Junquera, popular lugar para pasar el domingo, de degradación notable e impactos muy graves (basuras, lavado de coches...). En otros casos el bosque es intrincado y sombrío.

Contando con su interesante proximidad al casco urbano, podrían plantearse ambas márgenes como futuras zonas verdes de la ciudad, si bien esto requeriría de actuaciones cuantiosas dada la situación actual de deterioro.

Una vez en el caso urbano, la Huerva y sus riberas forman parte de parques urbanos como el Primo de Rivera y el Parque Bruil, precisamente un antiguo soto de este río.

\section{DIAGNOSTICO}

Los espacios naturales de ribera del término zaragozano presentan diversa extensión y grado de conservación y se encuentran bastante alejados entre sí, como prueba de su carácter relicto. No obstante, la densidad de vegetación natural es relativamente alta en comparación con otros tramos de las riberas del Ebro, Gállego o Huerva, máxime teniendo en cuenta la proximidad del núcleo urbano.

Ello supone un espacio natural muy interesante en conjunto, para el que hay que plantear las máximas medidas de protección con el fin de que no se pierda más terreno en lo que ya ha sido una drástica y dramática deforestación en las últimas décadas. Dada la 
complejidad e intensidad de los impactos, la recuperación de las riberas equiere de importantes esfuerzos, pero el valor potencial de estos espacios es enorme, de manera que una vez recuperados y eliminados los impactos principales, el mantenimiento no es muy costoso.

Los espacios seleccionados presentan entre sí una enorme heterogeneidad, tanto en lo referente a su valor, como a su grado de conservación y a sus posible usos:

a) Espacios en buen estado que deberían ser protegidos para preservar sus valores ecológicos: Soto de Mejana Redonda, Galacho de Juslibol y Soto de Partinchas, Sotos de Picatiel, Soto de las Perlas y Soto de Benedito en el Ebro, más el Soto de Aula Dei en el Gállego.

b) Espacios de interés pero que presentan impactos que requieren de corrección, planteándose una zonificación de usos como alternativa: Soto de Torre Alqué, Soto de Benavén, Soto de la Torre de Bergua, Soto de Cantalobos, Soto de Villarroya y Galacho de Lierta en las riberas del Ebro, además del conjunto de las riberas del Gállego.

c) Espacios muy degradados para los que se plantea básicamente una finalidad recreativa después de un adecuado acondicionamiento: Soto de Alfocea, Soto de la Almozara y Soto de La Cartuja en el Ebro, más el conjunto de las riberas de la Huerva.

\section{IMPACTOS}

El hombre ha preferido en las últimas décadas eliminar los sotos con el fin de ganar terreno para cultivar, requiriendo la construcción de costosas obras de defensa que han sustituido progresivamente a los bosques de ribera en la lucha frente a los caudales.

Esta deforestación ha sido el impacto básico, pero no debemos olvidar que la propia construcción de obras de infraestructura y defensa, especialmente dragados, también ha llevado consigo el deterioro o la eliminación de importantes masas arbóreas. 
Aun quedando reducidos a su mínima expresión, los sotos y riberas actuales siguen sufriendo impactos gravísimos, destacando:

-Extracciones de gravas, con sus consiguientes acumulaciones, que dada la proximidad de Zaragoza se han llevado a cabo intensivamente en las riberas del Ebro y del Gállego. Las alteraciones que producen estas extracciones son graves, pudiendo provocar modificaciones irreversibles, ya que se eliminan zonas de suelo que podrían soportar vegetación o cultivos, y los huecos resultantes pueden ser objeto de vertidos de residuos sólidos, con el consiguiente riesgo de contaminación en los acuíferos superficiales; además, pueden modificar el propio cauce y la dinámica fluvial.

-Escombros, vertidos y basuras dispersas, igualmente generalizados y en algunos casos abundantísimos (Soto de Alfocea, Soto de la Almozara, Soto de Cantalobos, riberas de Peñaflor).

-Presión de visitantes con una total falta de respeto hacia el medio (furtivismo, fuegos incontrolados, entresaca de ramas...).

-Elevada contaminación de las aguas.

Podemos hablar igualmente de un impacto institucional, referido a la falta de consideración hacia las riberas por parte de las instituciones y organismos públicos y privados a la hora de la ordenación, planificación y zonificación de usos de la llanura de inundación del río, de manera que no se tienen en cuenta en absoluto las bandas de servidumbre y policía que establece la Ley de Aguas.

\section{ALTERNATIVAS}

A pesar de su estado de degradación actual, la vitalidad ecológica de los sotos y su carácter pionero mantienen su posibilidad de pervivir. Las especies ribereñas gozan de una notable capacidad de regeneración, incluso después de ser tumbadas o arrancadas. Por ello, la recuperación de nuestras riberas no acarrearía en ningún caso cuantiosas inversiones. Es suficiente con permitir a la naturaleza su desarrollo y en dos o tres décadas se pueden cubrir las etapas hasta alcanzar el bosque climácico. 
Sería ideal poder contar con bosques ribereños continuos que jalonaran las orillas al menos entre el cauce y los diques de contención. Como primer paso hacia esa meta es preciso conservar los escasos retazos de soto que nos quedan estableciendo figuras de protección que zonifiquen los usos y eliminen los impactos.

Para llevar a la práctica esta necesidad se hacen imprescindibles las siguientes iniciativas:

-Dotación de una figura de protección global para el conjunto de sotos del Ebro y de las riberas del Gállego y Huerva además de otra concreta para cada uno de los espacios en función de su valor y situación actual, acompañada de una específica zonificación de usos.

-Realizar estudios globales y de temática específica para cada uno de los espacios y en función de ese conocimiento detallado de los mismos, valorarlos y jerarquizarlos de acuerdo con su interés ecológico. A partir de ahí se pueden planificar los distintos grados de protección y establecer medidas para alejar al gran público de los espacios más ricos acondicionando áreas de recreo en otros menos interesantes.

-Prohibición de toda actividad de extracción de áridos o vertido de escombros y, en su caso, obligación por parte de las empresas extractoras de restaurar la zona afectada.

-Aprovechamiento didáctico.

-Promoción de algunos espacios como área de recreo, con las medidas suficientes para evitar aglomeraciones y aumentar el conocimiento y el respeto hacia el medio:

-Evitar el acceso en coche a los espacios, estableciendo áreas de aparcamiento alejadas y promocionando el paseo a pie o en bicicleta.

-Establecer sistemas de vigilancia, especialmente en días festivos.

-Prohibir y sancionar todo tipo de vertido de basuras o escombros. Instalar contenedores y recoger las basuras con una periodicidad adecueda. 
-Elaboración de campañas de educación ambiental con itinerarios guiados por los distintos sotos, atendidos por monitores especializados y acompañados de folletos informativos.

-Establecer reservas integrales protegidas por barreras naturales, bien sotos completos, bien sectores de los mismos.
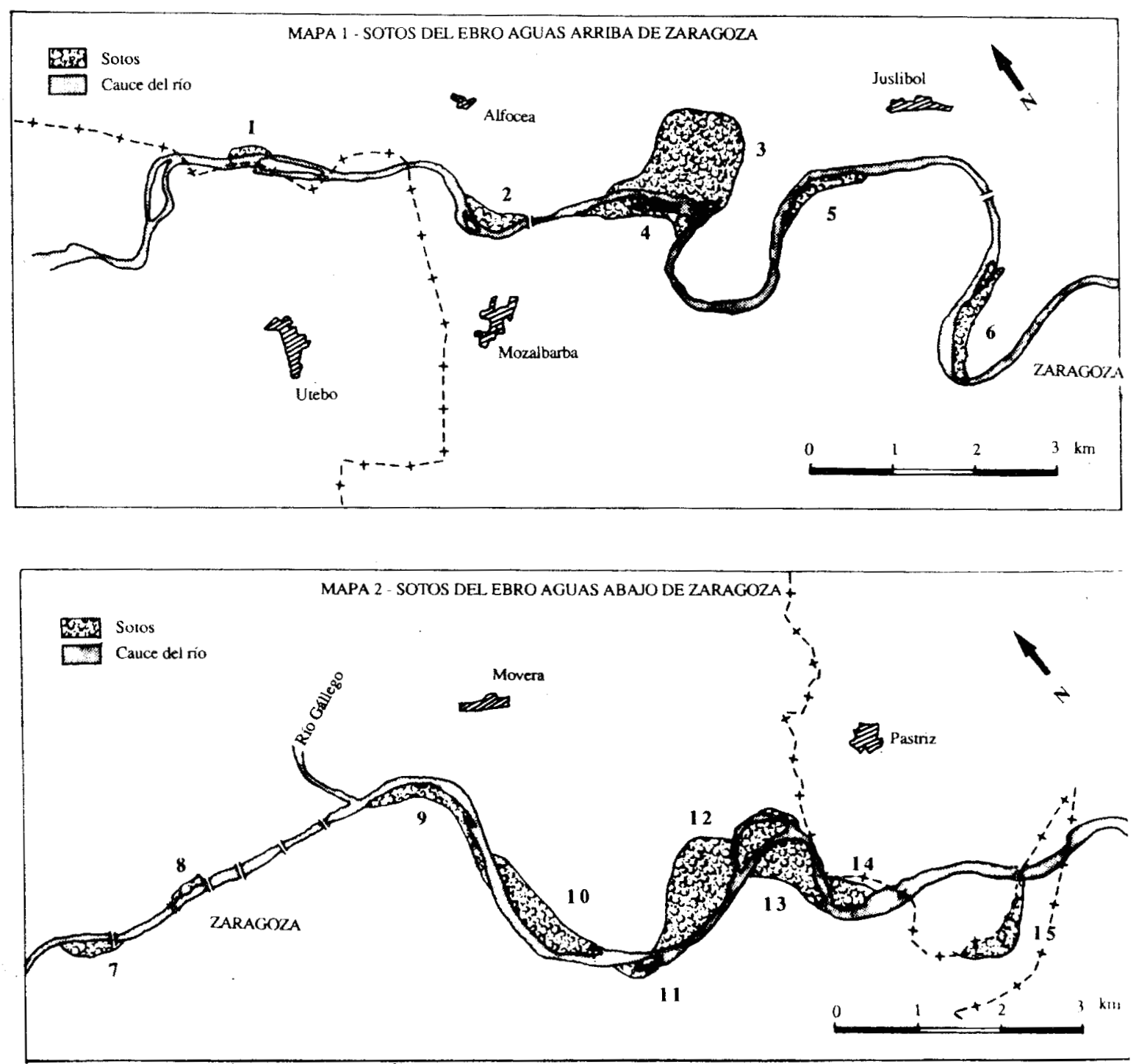


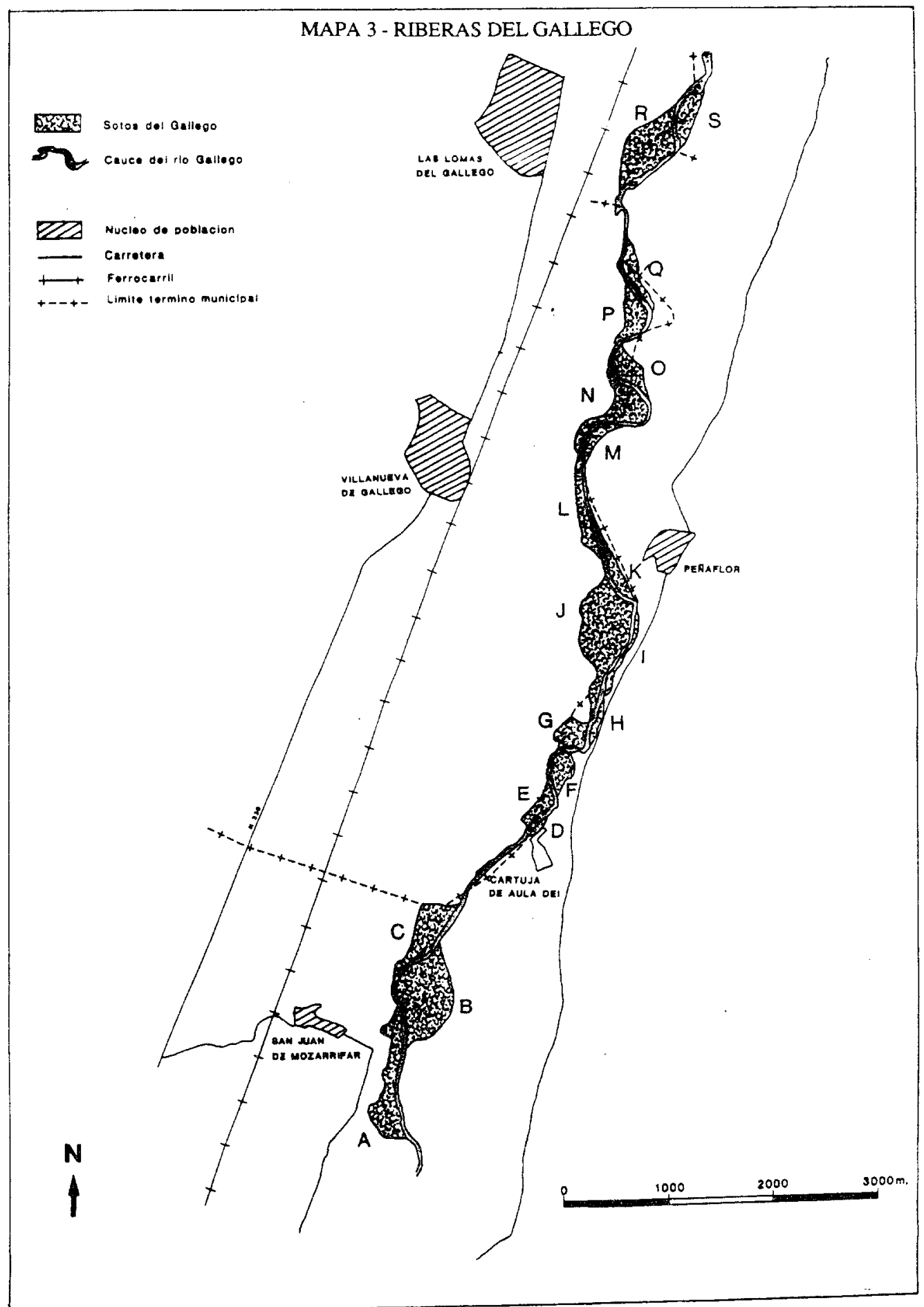




\section{9.- BIBLIOGRAFIA}

ANTON, A.; BALLESTER, C.; CABRERO, P.; OLLERO, A. y PELLICER, F. (1990): Inventario de Espacios Naturales del municipio de Zaragoza. Informe para el Servicio de Medio Ambiente del Ayuntamiento de Zaragoza, 311 págs. + mapa 1:50.000 (inédíto).

AYERRA, E. (1988): Estudio de los sotos de la Ribera tudelana. Servicio de Medio Ambiente. Diputación Foral de Navarra (inédito).

BOURRUT, H.; CONDE, O. y PELLICER, F. (1986): Aprovechamiento didáctico del Galacho de Juslibol y su entorno. Aspectos didácticos de Ciencias Naturales (Biología), 2, 31-58, 1.C.E. Universidad de Zaragoza.

GARCIA-ANQUELA, J.A.: TENA, J.M. y MANDADO, J.A. (1984): Las explotaciones de áridos como factor modificador de los cauces fluviales naturales. Cuadernos de Investigacion Geográfica. I Coloquio sobre procesos actuales en Geomorfologia, 83-89, Logrofio.

MONTSERRAT RECORDER, P.(1982): Aspectos ecológicos relacionados con la dinámica de sotos y riberas. Anales de Edafología y Agrobiología, XLI, 9-10, Madrid.

OLLERO OJEDA, A. (1989): Dinámica del cauce y de la llanura de inundación del río Ebro en el término de Alfaro (La Rioja). Cuadernos de Investigación Geografica, XV (1-2), 47-54, Logroño.

OLLERO OJEDA, A. (1989): Estudio ecogeográfico de los meandros del Ebro en el sector Rincón de Soto-Novillas. Memoria de Licenciatura (inédita). Departamento de Geografía, Universidad de Zaragoza, 454 págs. + cartografía.

OLLERO, A. y PELLICER, F. (1989): El impacto de las obras de defensa en los espacios naturales de la ribera del Ebro medio. Comunicaciones XI Congreso Nacional de Geografta, II, 412-422, Madrid.

PELLICER, F. y YETANO, M. (1985): El Galacho de Juslibol: un ejemplo de meandro abandonado. Cuadernos de Investigación Geográfica, XI, 113-124, Logronto.

REGATO PAJARES, P. (1988): Contribución al estudio de la flora y la vegetación del Galacho de la Alfranca en relación con la evolución del sistema fluvial. Diputación General de Aragón, 188 págs., Zaragoza. 